\title{
ANTROPOLOGÍA
}

\section{LA FAMILIA EN EL CONTEXTO DE LOS CUIDADOS HOSPITALARIOS. UNA PERSPECTIVA DESDE LA COMPETENCIA CULTURAL}

\author{
Miguel Ángel Fernández Molina
}

Doctor por la Universidad de Alicante. Director de Enfermería Hospital General

Universitario de Alicante (Departamento 19)

Profesor Asociado Departamento de Enfermería Universidad de Alicante

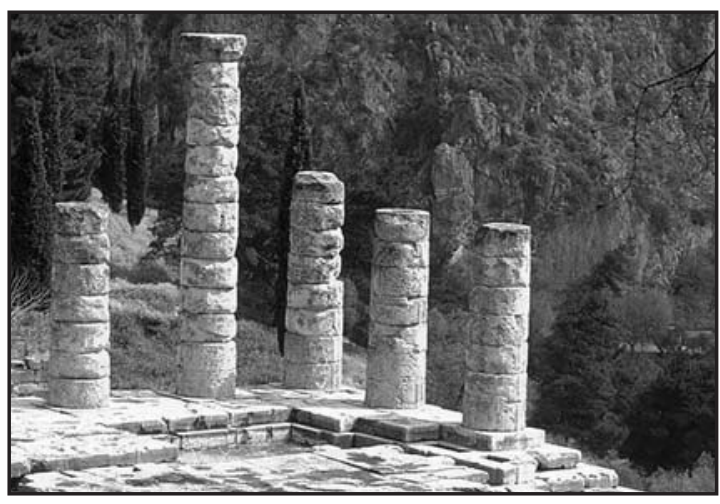

THE FAMILIY IN THE CONTEXT OF HOSPITAL CARE. A PERSPECTIVE FROM CULTURAL COMPETENCE

\section{SUMMARY}

$\mathrm{W}$

Te try to show the increasing role of family relatives during hospitalization and nursing initiatives for caring from a cultural competence perspective. In a health environment, it is considered as the ability of acting and obtaining positive clinical results during cross culture encounters. This paper not only focuses in diversity as a result of foreign population in Spain, even though $11.3 \%$ of population registered in Spain is foreign, and Alicante is the province with highest proportion (23.37\%). The case of Hospital General Universitario de Alicante is presented as a transferable experience to other hospitals and internal indicators demonstrate the enormous num- ber of personal interrelations that take place between the caring team and family relatives. Conclusions are reached and recommendations are proposed in order to obtain respect towards beliefs and values in patients and their family relatives. Together with other results, the discovery of a deep desire among professionals for acquiring cultural competence is relevant and so are figures such as percentage of complaint forms filled in and signed by family relatives.

Key words: Cultural competence, nursing, foreigners, family

\section{A FAMÍlIA NO CONTEXTO DOS CUIDA- DOS HOSPITALARES: UMA ANÁLISE SOB A PERSPECTIVA DA COMPETENCIA CUL- TURAL}

\section{RESUMO}

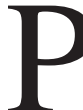

retende-se mostrar a função crescente da família durante a hospitalização, bem como as iniciativas da Enfermagem para cuidar desde a perspectiva da competência cultural. No mundo sanitário, a habilidade de atuar e obter resultados clínicos positivos nos encontros entre culturas distintas mostra-se cada vez mais freqüente.Assim, este trabalho centra-se não somente na diversidade da população estrangeira na Espanha, embora $11,3 \%$ da população radicada na Espanha seja estrangeira, e Alicante é a província com a 
maior proporção $(23,37 \%)$. Apresenta-se o caso do Hospital Geral Universitário de Alicante como exemplo, cuja experiência é transferível a outros hospitais, tendo em vista que seus indicadores assistenciais evidenciam o crescente número de relações interpessoais, as quais se produzem entre a equipe de cuidadores e a família. Tal fato possibilita uma série de conclusões e permite formular recomendações para alcançar o respeito às crenças e valores de pacientes e seus familiares. Destaques incluem a descoberta entre os profissionais de um profundo desejo de adquirir competências culturais, bem como dados como a percentagem de folhas de reclamações assinadas pelos membros da família.

\section{Palavras-chave: Competência Cultural;} Enfermagem; Estrangeiros; Família

\section{RESUMEN}

Ne pretende mostrar el rol creciente de la familia durante la hospitalización así como las iniciativas de enfermería para cuidar desde la perspectiva de la competencia cultural. En el mundo sanitario, es la habilidad de actuar y obtener resultados clínicos positivos en los encuentros entre culturas distintas. El trabajo no sólo se centra en la diversidad fruto de la población extranjera en España, aunque el 11,3 \% de la población empadronada en España es extranjera, y Alicante es la provincia con la mayor proporción $(23,37 \%)$. Se presenta el caso del Hospital General Universitario de Alicante como ejemplo cuya experiencia es transferible a otros hospitales y a través de sus indicadores asistenciales se evidencia el ingente número de interrelaciones personales que se producen entre el equipo cuidador y la familia. Se alcanzan una serie de conclusiones y se formulan recomendaciones para alcanzar el respeto a las creencias y valores de pacientes y sus familias. Destacan, entre otros, el hallazgo entre los profesionales de un profundo deseo de adquirir competencia cultural al igual que datos como el porcentaje de hojas de quejas firmadas por familiares.

Palabras clave: Competencia cultural, enfermería, extranjeros, familia

\section{INTRODUCCIÓN: EL NUEVO CONTEX- TO HOSPITALARIO}

"La organización de todos los recursos y dispositivos asistenciales garantizará la cobertura sanitaria a toda la población según criterios objetivos que contemplen factores geográficos, demográficos, epidemiológicos, socioeconómicos y culturales" (Decreto 74/2007). Que mejor forma de mostrar la actual sensibilidad de la sociedad en general y de las autoridades sanitarias en particular hacia las cuestiones culturales que incorporar a la legislación sanitaria estos aspectos a pesar de la tremenda complejidad que supone la gestión clínica y la provisión de cuidados en los centros asistenciales.

Los modernos hospitales de agudos son considerados verdaderos "centros de alto rendimiento" donde trabajan profesionales integrados en equipos también de alto rendimiento. En la práctica supone atender a un número cada vez mayor de pacientes y, a ser posible, en el tiempo más corto posible y con la máxima calidad. Un indicador de referencia como es la "estancia media" no es otra cosa que "el tiempo -expresado en días- que tardamos en resolver el problema al paciente". Ese tópico de que la sociedad cambia cada vez más deprisa tiene su reflejo en el mundo hospitalario y, para los que en él trabajamos, es además un hecho constatado.

A través de un ejemplo real, y cuyo escenario pensamos es transferible a la mayoría de los centros sanitarios públicos del sistema de salud, se pretende mostrar una visión actual de este nuevo contexto. Para ello, se aportan los datos del año 2007 en el Hospital General Universitario de Alicante (HGUA) (SIG, 2007) como ejemplo de ese rendimiento y para así estimar el número de interrelaciones personales que se producen en un hospital. Los más relevantes para comprender el elevado número de encuentros entre pacientes/familia y profesionales serían:

- Ingresos totales: 33.870

- Estancias: 243.357

- Estancia Media: 7,19

- Visitas en Consultas Externas: 270.380

- Visitas en Centro de Especialidades: 243.475

- Urgencias Atendidas: 168.687

- Intervenciones Quirúrgicas: 26.555

De entre toda esa actividad, se estima que el número de pacientes extranjeros ingresados se 


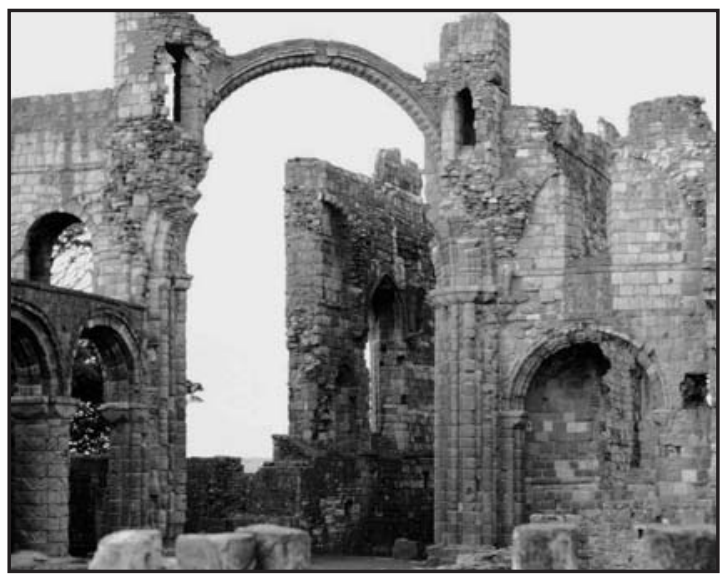

sitúa entorno al $8 \%$ (siendo el porcentaje de residentes extranjeros el 23,37\% en la provincia). Mi actual aportación no pretende centrarse exclusivamente en los pacientes extranjeros y sus familias por una razón: si bien pueden haber casos aislados en extranjeros, en la práctica nos encontramos principalmente "choques culturales" con pacientes y familias no extranjeras motivados por: condición religiosa (p.ej. en Testigos de Jehová), cuestiones costumbristas (p.ej. en etnia gitana) o cuestiones sociales (p.ej en personas inadaptadas socialmente de trato conflictivo con el personal).

Frente a la hospitalización convencional (la clásica de toda la vida, donde por general el paciente y su familia vivían durante un tiempo más o menos prolongado la vida en el hospital) ahora la mayoría de estos se benefician de lo que se vienen a denominar "alternativas a la hospitalización convencional": Hospitales de Día, Cirugía sin Ingreso, Unidad de Hospitalización a Domicilio (UHD), Unidades de Corta Estancia, Consultas de Alta Resolución, etc. En el caso de la UHD, el profesional desarrolla su labor no ya en el hospital sino inmerso plenamente en el contexto familiar del paciente: en su hogar y rodeado de sus allegados. Al reducirse en los Hospitales de Agudos el tiempo de "exposición" en el seno de ese triángulo formado por paciente, familia y personal sanitario, se podrá entender que cobre una importancia notable el que el encuentro se produzca de la manera más satisfactoria ya que no se cuenta con margen suficiente para "enderezar" una mala relación. Aquí, el minuto de oro (ese primer contacto que va a marcar la relación posterior con signo positivo o nega- tivo) debe tenerse más en cuenta incluso que en otras situaciones asistenciales.

Aquellos pacientes que no son subsidiarios de beneficiarse de estas alternativas, ingresan en el centro sanitario en camas convencionales (o de críticos) también con una disminución global del tiempo de hospitalización, pero con más posibilidad de que se desarrollen fenómenos de convivencia entre paciente, familia y personal sanitario. Más tiempo para que sean fructíferos, .... o más tiempo para que se deterioren.

\section{COMPETENCIA CULTURAL EN EL CONTEXTO HOSPITALARIO}

Es un hecho que las enfermeras en nuestros hospitales han pasado de cuidar a sus familiares, amigos, vecinos y paisanos a cuidar a personas de costumbres y creencias con frecuencia muy diferentes a los suyos. La competencia cultural en el mundo sanitario es la habilidad de actuar y obtener resultados clínicos positivos en los encuentros entre culturas distintas.

Existen múltiples definiciones de competencia cultural. Estas han evolucionado desde perspectivas, intereses y necesidades diferentes, y se han incorporado a las leyes de los estados, los reglamentos (ya se ha citado en la introducción la alusión a factores culturales que recoge el Reglamento publicado en el DECRETO 74/2007 de la Conselleria de Sanitat de la Comunidad Valenciana), programas sanitarios, las organizaciones del sector privado y el ámbito académico. De todas las definiciones que existen, podríamos elegir la que preconiza desde 1994 el Seattle King Country Department of Public Health, por contener todos los términos que utiliza el lema de las VI Jornadas Internacionales de Cultura de los Cuidados:

COMPETENCIA CULTURAL: "Habilidades de individuos y sistemas de responder respetuosa y eficazmente a las personas de todas las culturas, clases, etnias y religiones de una manera que reconoce, afirma y valora las diferencias culturales y similitudes, y el valor de los individuos, familias y comunidades, y protege y conserva la dignidad de cada uno" 
¿Que supone en la práctica este paradigma en la forma de cuidar? Pues la competencia cultural requiere conocimientos, habilidades y actitudes a la hora de dar cuidados a diferentes grupos de pacientes y familias. Como se puede observar, no sólo requiere conocimientos culturales sino también hace referencia a la práctica y el comportamiento de los profesionales de los cuidados. Así pues, la enfermera culturalmente competente tendrá tres habilidades cuando se encuentre con una familia de cultura diferente: conocimientos, habilidades y actitudes, dicho de otro modo: Saber, Saber Hacer y Saber Estar.

Aunque tímidamente, comienza a detectarse una creciente preocupación en nuestro contexto por evaluar la confianza que tienen las enfermeras españolas a la hora de atender a pacientes -y sus correspondientes familias- de grupos culturales diversos y así desarrollar programas de formación pre-grado que contemplen la administración de cuidados culturalmente sensibles (Vargas et al., 2004). Asimismo, conviene señalar la iniciativa de la Generalitat de Catalunya al editar su "Guía para el respeto a la pluralidad religiosa en el ámbito hospitalario", conscientes de vivir una realidad de diversidad religiosa creciente merced a la inmigración a pesar de considerarse la sociedad catalana como secular (Generalitat de Catalunya, 2005).

Aquí surge una nueva pregunta. Las autoridades sanitarias y el mundo universitario parecen estar cada vez más concienciados, ¿pero realmente desean o sienten la necesidad las enfermeras de adquirir competencia cultural?

De entre los datos obtenidos con motivo de la tesis doctoral "Estudio de los cuidados a pacientes extranjeros hospitalizados desde la perspectiva de la Enfermería Transcultural" (Fernández, 2006) en el HGUA a través de una encuesta realizado a 40 profesionales se comprobó lo siguiente:

- 81,25\% nunca preguntaban la religión a la hora de cumplimentar la Hoja de Valoración del paciente al ingreso.

- Sin embargo, el 87,5 siempre lo preguntaban a la hora de pedir la dieta.

- En cuanto a consecución de auxilio espiritual específico variaba entre $31,25 \%$ casi nunca, $25 \%$ algunas veces, siempre y nunca el $12,5 \%$ ambos.
- La preservación, acomodación o remodelación de los cuidados obtuvo una mayoría de frecuentemente $(25 \%)$ repartiéndose el resto de valores en porcentajes del 18,75\% respectivamente.

Pero los datos más relevantes para la temática que pretendemos abordar fueron las siguientes:

- La mayoría (87,5\%) estaban abiertos a la comprensión de las tradiciones de salud.

- El 93,75\% afirmaron prestar atención a la comunicación con los extranjeros.

- El $68,75 \%$ implicaba a la familia en el proceso de cuidar.

- Y finalmente, el 87,5\% de encuestados consideraban que los factores culturales eran relevantes para la salud, enfermedad y a la hora de administrar los cuidados.

\section{PACIENTE Y FAMILIA.}

"NO INGRESA UN PACIENTE, INGRESA UN MIEMBRO DE UNA FAMILIA". Puede parecer una frase hecha, pero lo cierto es que desde siempre la familia del paciente ha tenido un protagonismo relativo, que ha ido aumentado en paralelo a los cambios sociales que todos vamos experimentando. La sola presencia de un familiar puede desencadenar en el personal sanitario una serie de reacciones "a priori". Como sabemos, el estereotipo se basa en juzgar a alguien sobre la base al "grupo" al que pertenece, pudiendo surgir distorsiones que dan lugar a prejuicios sobre: razas, nacionalidades, creencias, etc.

La experiencia acumulada en la práctica diaria en los centros asistenciales nos llevan a afirmar que la familia en nuestros países latinos y católicos (de Europa y de América) y a diferencia de la anglosajona y protestante, es muy paternalista/proteccionista y ello genera frecuentes problemas en la atención a los enfermos. Un conflicto muy frecuente, casi diario, es la demanda por parte de los familiares de que ocultemos al enfermo el diagnóstico y el pronóstico. Otro riesgo es cuando existe disparidad de criterios entre el paciente, los familiares y el equipo terapéutico, siendo un verdadero esfuerzo alcanzar el necesario acuerdo. Sin el acuerdo de los familiares, por ejemplo en caso de cuidados paliativos, se pueden acarrear problemas importantes durante el episodio asistencial. A los familiares los 
necesitamos como aliados y no como enemigos. El tiempo que dediquemos a los familiares siempre será un tiempo muy provechoso, por el bien de los familiares y también por el del propio paciente (Ética en los cuidados paliativos, 2006)

No sólo los vínculos familiares influyen, incluso podría ir más lejos y añadir que, además, ingresa un miembro real o potencial de una asociación de afectados que también puede tener su influencia. Seguro que muchos de los presentes con actividad asistencial son conscientes del peso y el poder de determinadas familias de pacientes y las asociaciones que las acogen. Con todos ellos interactúa el profesional de enfermería.

Todos sabemos que escuchar a la familia forma parte de la primera obligación del profesional sanitario: conocer los hechos que, una vez esclarecidos, permiten identificar los valores en conflicto, condición previa a la búsqueda de la solución al problema del paciente Además, no es difícil estimular a la familia, y en especial al cuidador principal, a que formulen las dudas y preocupaciones que les surjan.

Como muestra palpable de esta afirmación, quisiera llamar la atención acerca de las quejas y reclamaciones tramitadas en nuestro hospital, ya que del total de 959 quejas escritas registradas en el Servicio de Atención e Información al Paciente (SAIP) desde el 1 de enero de 2007 al 7 de abril de 2008, 289 quejas -o lo que es lo mismo el $30 \%$ fueron realizadas por la familia (no por el paciente al que se le atribuye clásicamente el protagonismo absoluto dentro del sistema sanitario).

Añadir, que incluso las encuestas de satisfacción que se realizan sistemáticamente en los centros sanitarios de la Agencia Valenciana de Salud tienen preguntas dirigidas al familiar, no sólo al paciente. Por todo ello, no es baladí otorgar a la familia el protagonismo que viene reclamando, ya que su poder de influencia sobre el sistema es significativo.

\section{SITUACIONES DE PROTAGONISMO FAMI- LIAR}

De forma breve, y a modo de ilustración, paso a enumerar algunos de los casos que acontecen en un hospital donde la familia tiene especial protagonismo y donde la competencia cultural se requiere para una buena relación:
- En todas las unidades pediátricas. (Además, Asociaciones como ASPANION han negociado mejoras como la cama del familiar y la dieta al acompañante del niño oncológico o con enfermedad crónica ingresado).

- En unidades con pacientes en situación crítica (UCI, Reanimación, Quemados).

- En unidades con pacientes con elevada dependencia de cuidados.

- Con motivo de Consentimiento para la Donación de órganos. Aquí la especialización en la aproximación a la familia del donante convierte al HGUA en referencia mundial y con amplia repercusión mediática.

- En unidades con pacientes y familias cuyas costumbres difieren del profesional. Sin pretender caer en estereotipos, ejemplos como:

- Acompañamiento familiar en etnia gitana

- Acompañamiento familiar en nórdicos

- Acompañamiento del esposo árabe en el paritorio

- Rituales en recién nacidos de origen Subsahariano

- Preceptos en Testigos de Jehová

- Costumbres culinarios (ej. el conejo es considerado animal de compañía en los nórdicos por lo que lo rechazan como alimento)

\section{CONCLUSIONES}

Recomendaciones para la Competencia Cultural en el contexto de los cuidados hospitalarios.

\section{MINUTO DE ORO.}

$\mathrm{Al}$ iniciar nuestras relaciones profesionales con los pacientes y sus familias debemos de ser conscientes de la importancia del "primer contacto" ya que puede condicionar el resto del episodio asistencial

\section{INICIAR EL PROCESO DE ATENCIÓN DE ENFERMERÍA CON UNA CORRECTA VALO- RACIÓN.}

Para poder planificar adecuadamente cuidados congruentes con la cultura del paciente, se hace necesario que la primera etapa del proceso enfermero contemple la valoración de los aspectos básicos inherentes a la cultura del mismo. 


\section{GUARDAR RESPETO A LOS VALORES DEL OTRO.}

El paciente y su familia tienen derecho reconocido a que sus creencias, valores y costumbres sean respetados.

\section{UNA PRÁCTICA ASISTENCIAL "TRANS- CULTURAL" AMORTIGUA LAS DESIGUAL- DADES DE SALUD.}

La mejor garantía de una equidad en la asistencia es el respeto individualizado que propugna la Enfermería Transcultural.

\section{EVITAR ESTEREOTIPOS Y ETIQUETAS CULTURALES}

Muchas veces somos los propios profesionales e investigadores los responsables de crear etiquetas para fundamentar nuestras teorías.

\section{LIDERAZGO DE ENFERMERÍA EN LAS} POLÍTICAS DE FORMACIÓN MULTICULTU-

\section{RAL PARA PROFESIONALES.}

Por su cercanía al paciente y su entorno familiar, unidos a la creciente vocación transcultural, es la/el enfermera/o el profesional mejor posicionado actualmente para formar a otros miembros del equipo multidisciplinar en competencia cultural.

7. ADAPTAR CULTURALMENTE EN LOS CENTROS SANITARIOS LA ASISTENCIA, ALIMENTACIÓN Y LOS ESPACIOS DE DUELO Y CULTO.

Dentro de la Misión de los Centros Asistenciales, debería figurar esta sensibilidad cultural y manifestarlo por escrito.

\section{NO AL ETNOCENTRISMO Y AL CULTURA- LISMO.}

Los profesionales formamos grupos con nuestros propios valores que imponemos al resto de pacientes. El etnocentrismo característico de nuestro sistema sanitario nos hace sentirnos cómodos y caemos en el culturalismo (la comparación con los "otros": el "nosotros" frente a "ellos").

\section{GUÍAS DE ACTUACIÓN DE ENFERMERÍA DE COMO PRESERVAR, ACOMODAR Y REMODELAR LOS CUIDADOS.}

Facilitar a los profesionales herramientas que les permitan trasladar al enfermo y a su familia la percepción de que somos competentes culturalmente, y capaces de ser "pro-activos" en lugar de "reactivos" en el respeto cultural.

\section{FAMILIA INFORMADA CONTÍNUAMENTE.}

Aunque a veces la prudencia del paciente o familia (frecuente en extranjeros principalmente nórdicos) pueda interpretarse como falta de interés por conocer su evolución, se ha constatado un déficit evidente en la información que reciben.

\section{LA COMPETENCIA CULTURAL CONFIE-} RE AL PROFESIONAL UN VALOR AÑADIDO A SU AUTONOMÍA Y PRESTIGIO

Debemos recordar que la "competencia cultural" (es decir, la habilidad de actuar y obtener resultados clínicos positivos en los encuentros entre culturas distintas) supone para el profesional un paso cualitativo en su desarrollo profesional y una ocasión para las autoridades sanitarias de ofrecer un servicio de salud en verdad "equitativo y universal".

\section{BIBLIOGRAFÍA}

- Guías de Ética en la práctica médica. (2006) Ética en cuidados paliativos. Edita Fundación de Ciencias de la Salud. Madrid. - Fernández Molina, M.A. (2006) Antropología de los Cuidados. Estudio de los cuidados a pacientes extranjeros hospitalizados desde la perspectiva de la Enfermería Transcultural. Edita: Consejo de Enfermería de la Comunidad Valenciana (CECOVA). $\mathrm{N}^{\mathrm{o}}$ 50. Alicante.

- Generalitat de Catalunya. (2005) Guia per al respecte a la pluralitat religiosa en 1'àmbit hospitalari, Departament de la Presidencia, Direcció General d’Afers Religiosos, Departament de Salut.

- Vargas Jiménez, J.A., Molina Contreras, J.L., Shellman, J. y Bernal, H. The confidence of Spanish nurses in caring for culturally diverse groups, Alicante, III Jornadas Internacionales de Cultura de los Cuidados \& 30th Annual Conference of the Transcultural Nursing Society y VI Reunión sobre Investigación Cualitativa en Salud, 14-16 junio 2004, Libro de Abstracts, (Comunicación).

\section{OTRAS FUENTES}

- DECRETO 74/2007, de 18 de mayo, del Consell, por el que se aprueba el Reglamento sobre estructura, organización y funcionamiento de la atención sanitaria en la Comunidad Valenciana. Artículo 3.3.

- Instituto Nacional de Estadística (INE) Avance del Padrón Municipal a 1 de enero 2008.

- Servicio de Atención e Información al Paciente (SAIP) del Hospital General Universitario de Alicante del Departamento de Salud 19. Quejas escritas registradas entre 01-01-2007 y 07-04-2008.

- Sistema de Información para la Gestión (SIG) 2007 elaborado por la Unidad de Admisión del Hospital General Universitario de Alicante del Departamento de Salud 19. 2007 